[Aus dem Hygienischen Institut der Universitāt Halle.]

(Stellvertr. Direktor: Privatdozent Dr. W. Schürmann.)

\title{
Bakteriologische Untersuchungen über eine neue Methode der Händedesinfektion mit Ausschaltung von Seifenwaschung nach Gocht. Von
}

Karl Stahlschmidt.

Vor einiger Zeit erschien in der Deutschen Medizinischen Wochenschrift eine kurze Mitteilung von Gocht über Händedesinfektion ohne Seife. Dieses neue Desinfektionsverfahren lehnt sich an die Ahlfeldsche Heißwasser-Alkohol-Methode an, unterscheidet sich jedoch darin von letzterer, daß statt der Seife feines Alabastergipspulver angewendet wird. Der Vorgang bei dieser Art der Händedesinfektion ist der, daß nach Anfeuchtung beider Hände Handrücken und Handflächen in das Gipspulver getaucht und dann gegenseitig gewaschen werden, genau so, als ob man eine Seifenwaschung vornähme; wird eine Bürste benutzt, so wird auch sie angefeuchtet in den Gips getaucht und mit dem daran hängenbleibenden Pulverbrei Hände und Unterarme gebürstet. Die ganze Prozedur wird unter einem dünnen Strahle warmen Wassers vorgenommen. Nach 10 Minuten ləngem Waschen werden die Gipsreste abgespült. Nach dieser mechanischen Reinigung werden Hände und Unterarme einige Minuten lang mit 70 proz. Alkohol und einem sterilen Tupfer abgerieben. Das ist in kurzem die von Goch t empfohlene Desinfektionsmethode.

Gocht legt noch bei aseptischen . Operationen sterilisierte Zwirnhandschuhe an, während die. Assistenten und die Operationsschwester, die. auf dieselbe Art und Weise wie der Operateur ihre Hände desinfizieren, ohne Handschuhe arbeiten.

Am Schlusse der Mitteilung wird von Gocht darauf hingewiesen, dab sich seine Methode bei allen vorgenommenen Operationen glänzend bewährt 
hat, und bisher nur gute aseptische Wundheilungen zu verzeichnen gewesen sind. Ein in Kriegszeiten nicht $\mathrm{zu}$ unterschätzender Vorzug dieser Methode der Händedesinfektion ist der, daß dieselbe die Seife erspart, daß wir in dem Gipspulver ein Reinigungsmittel haben, das die Haut nicht angreift, das außerdem überaus billig und stets leicht $\mathrm{zu}$ beschaffen ist.

Vor Besprechung der bakteriologischen Versuche und Untersuchungen über die Gochtsche Händedesinfektion wären noch einige Punkte von grundlegender Bedeutung zu erörtern. Vor allem muß hervorgehoben werden, daß es sich bei dieser neuen Methode nur um eine rein chirurgische Händedesinfektion handelt, nicht aber auch um eine hygienische, denn mit der Gipswaschung allein - soviel will ich gleich vorausnehmen - wird eine Keimabtötung nicht erzielt, und so würde denn besonders beim Gebrauche einer Bürste eine bedeutende Verstreuung und Weiterverbreitung der an die Hände gelangten Krankheitskeime stattfinden, und neue Infektionsquellen würden geschaffen werden, ein Faktor, auf den bei der hygienischen Desinfektion besonderes Gewicht gelegt werden muß. Aus diesem Gesichtspunkte heraus fordert auch Flügge als erste Waschung nach einer Händeinfektion eine solche mit einer keimvernichtenden Flüssigkeit, woran dann eine mechanische Reinigung angeschlossen werden kann. Dieses Postulat wird durch die Gips-Alkohol-Waschmethode nicht erfüllt.

Entsprechen die Untersuchungen im bakteriologischen Laboratorium den oben erwähnten ausgezeichneten Resultaten der Praxis? Um diese Frage richtig beantworten zu können, zog ich die bakteriologischen Untersuchungsbefunde der von der überwiegenden Mehrzahl der Operateure geübten Händedesinfektionsverfahren zum Vergleiche heran. Dabei ging ich sicher in der Annahme nicht fehl, daß die etwas modifizierte HeißwasserAlkohol-Methode Ahlfelds, von Herff einst stark befürwortet, die am weitesten verbreitete und bevorzugte dieser Art ist. Bekanntlich besteht sie darin, daß die Hände bei Gebrauch eines Nagelreinigers mit einer Bürste gründlich ungefähr 10 Minuten lang unter fließendem heißen Wasser abgeseift und darauf 5 Minuten mit Alkohol unter Benutzung einer sierilisierten Bürste oder eines Tupfers gründlich abgerieben werden.

In der diesbezüglichen Literatur fand ich die einander z. T. widersprechendsten bakteriologischen Resultate, wofür in erster Linie das Moment -als das ursäcbliche anzusprechen ist, daß sich fast jeder, der das Ahlfeld sche Desinfektionsverfahren auf seine Brauchbarkeit hin bakteriologisch untersucht hat, einer eigenen von der der anderen abweichenden Versuchsmethode und Anordnung bedient hat. Der Grund hierfür ist der, daß alle bestrebt waren, die Art und Weise der Keimentnahme von der Hand als 
den wesentlichsten Gesichtspunkt bei einer derartigen Untersuchung so zu gestalten, da $B$ möglichst viel Keime von der auf ihren Bakteriengehalt zu prüfenden Handoberfläche in die Nährflüssigkeit oder den Nährboden übergingen, und so möglichst genaue Befunde erhoben werden konnten. Während Ahlfeld selbst mit einem sterilen Stäbchen den Unternagelraum und das Nagelbett eines einzigen Finger auskratzte und dann das Stäbchen in Nährbouillon warf, führte Fürbringer dieselbe Manipulation an allen fünf Fingern aus und benutzte außerdem flüssig gemachte Gelatine, so daß er nachher unter. Umständen nicht nur das Vorhandensein, sondern auch die Zahl der Keime bestimmen konnte. Andere Untersucher drückten ihre Hände in erstarrten Agar oder Gelatine. Auch die weitere Behandlung dieser beimpften Nährsubstrate war nicht einheitlich, z. T. wurden sie bei Zimmertemperatur aufbewahrt, z. T. im Brutschrank. Alle diese Differenzen in der Abimpfungstechnik usw. machten es mir wegen ihrer verschiedenen Untersuchungsbefunde unmöglich, die letzteren zum Vergleiche verwerten zu können. Bevor ich über den Ausfall meiner Untersuchungen berichte, möchte ich die meinen Versuchen zugrunde liegende Abimpfungstechnik kurz schildern.

Filtrierter Agar und zwar 40 bis $50 \mathrm{ccm}$ in Erlenmeyerkölbchen wurden im Wasserbade flüssig gemacht, dann auf $45^{\circ}$ abgekühlt. Sollte eine Abimpfung der Hände viorgenommen werden, so wurde von dem Laboratoriumsdiener aus einem gebrauchfertigen Kölbchen, dessen Rand in der Bunsenflamme abgeglüht worden war, der flüssige Agar langsam in dünnem. Strahle über den Handrücken und in die hohle Hand gegossen und dabei mit der betreffenden Hand Waschbewegungen gemacht, wobei besonders der Daumen kräftig die Nagelfalze der übrigen vier Finger abrieb und die Fingerbeeren von den Nagelenden abzog, so daß der Agar ausgiebig durch die subungualen Räume hindurchfließen konnte. Der von der Hand und durch die Fingerzwischenräume abfließende Agar wurde in sterilen Petrischalen aufgefangen. Bei nötiger Übung und richtiger Konsistenz des Agars blieb stets nur ein ganz dünner Uberzug von demselben an der Hand zurück, der auf weiter unten zu besprechende Art und Weise je nach der Art des Versuches wieder entfernt wurde. Für jede Hand wurde der Inhalt eines halben Kölbchens (etwa $25 \mathrm{ccm}$ ) gebraucht: Ich glaube, daß bei dieser Art des Abspülens der Hände in allen ihren Teilen mit flüssigem Agar eine ganz besonders ausgiebige thertragung der Hautkeime auf den Nährboden erreicht wurde. Probeuntersuchungen ergaben, daß die ausgekratzten Unternagelräume und Nagelfalze im Vergleich zu der auf diese Art und Weise gewonnenen Keimmengen nur ganz minimale Keimzahlen lieferten. Die beimpften Nährböden kamen in den Brutschrank von $37^{\circ}$. 
Die Resultate, die sich bei den Desinfektionsversuchen ergaben, habe ich in Tabellen zusammengestellt. Bei Angaben der Anzahl der Kolonien bedeutet der Ausdruck ,unzählige“" mehr als 20000, mehr als 10000 soll besagen, daß zwar eine genaue Zählung, wie es ja bei den kleinen Petrischalen trotz Vergrößerung selbstverständlich ist, nicht möglich war, daß aber mit großer Sicherheit die Schätzung höchstens 20000 Kolonien ergab.

Bei jeder Versuchsperson sind die Ergebnisse jeder Hand gesondert angegeben. Wenn ich mir auch bewußt bin, daß natürlich bei dem ganzen Desinfektionsvorgang eine Keimübertragung von der einen Hand auf die andere leicht stattfinden konnte, so glaube ich doch berechtigt zu sein, jedesmal zwei gesonderte Versuche annehmen zu dürfen. Denn erstens ist die Anzahl der übertragenen Keime sicher nur sehr gering und spielt daher bei den sonstigen hohen Zahlenwerten nur eine ganz untergeordnete Rolle, und zweitens wurde nach der Alkoholwaschung - aber auch schon nach Möglichkeit während derselben - streng darauf geachtet, daß eine gegenseitige Berührung beider Hände nicht erfolgte.

Die Händedesinfektion nach der modifizierten Ahlfeldschen Methode gestaltete sich dementsprechend folgendermaßen:

Vor der Waschung wurden bei jeder Versuchsperson beide Hände jede für sich - abgeimpft, um ein annäherndes Bild von ihrem oberflächlichen Keimgehalt zu gewinnen. Dann erfolgte die 10 Minuten lange Waschung unter fließendem Wasser, dessen Temperatur 35 bis $40^{\circ}$ betrug, mit Seife und Bürste. Während der Waschung wurden die Unternagelräume mit einem NageIreiniger ausgekratzt. Hieran schloß sich eine zweite Abimpfung, ohne daß die Hände abgetrocknet worden waren. Die den Fingern anhaftenden Spuren von Agar wurden mit Leitungswasser kurz abgespült, und nun wurden die Hände mit Alkohol mittels eines sterilen Tupfers 5 Minuten lang intensiv abgerieben. Nach Abtrocknen der Hände mit einem sterilen Mulläppchen wurde schließlich ihr nunmehriger Keimgehalt durch Plattengießen festgestellt: Bei dieser Desinfektion benutzte ich gewöhnliche Kernstückseife anstelle der sonst üblichen Schmierseife. Bürsten und Nagelreiniger waren durch Auskochen steril gemacht worden und befanden sich nicht, wie es z. T. üblich ist, in irgendeiner Desinfektionsflüssigkeit, um nicht durch die gleichzeitige Anwesenheit auch noch so geringer Mengen einer derartigen Lösung die alleinige Wirkungsweise des zu untersuchenden Desinfizienz zu verändern.

Betreffs des zu verwendenden Alkohols entschied ich mich, da ịm allgemeinen sowohl 60 prozentiger als auch absoluter Alkohol und die dazwischenliegenden Konzentrationswerte bei der Händedesinfektion in Anwendung kommen, für einen mittelwertigen und zwar 70prozentigen Alkohol. Hierbei 
möchte ich von vornherein bemerken, daß alle Prozentzahlen für Alkohol von mir nach Volumprozent und nicht Gewichtprozent gerechnet werden. Im ïbrigen soll auf die Desinfektionskraft der verschiedenen Alkoholkonzentrationen weiter unten näher eingegangen werden.

Die nun folgende Tabelle 1 enthält die bakteriologischen Ergebnisse der Händedesinfektion durch Abseifung (Kernseife) und nachfolgende Alkoholspülung.

Tabelle 1.

\begin{tabular}{|c|c|c|c|c|c|c|c|c|c|c|c|}
\hline \multirow[b]{2}{*}{ Nr. } & \multirow[b]{2}{*}{ Datum } & \multirow[b]{2}{*}{ Person } & \multirow{2}{*}{$\begin{array}{l}\text { Kolonien } \\
\text { von der } \\
\text { Tageshand } \\
\text { vor der } \\
\text { Desinfektion }\end{array}$} & \multicolumn{3}{|c|}{ Seifenwaschung } & \multicolumn{3}{|c|}{$\begin{array}{c}70 \% \text { Alkohol- } \\
\text { waschung }\end{array}$} & \multirow[b]{2}{*}{ 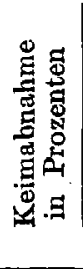 } & \multirow{2}{*}{ 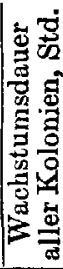 } \\
\hline & & & & 吾 & 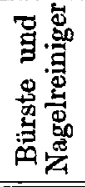 & $\begin{array}{c}\text { Gewachsene } \\
\text { Kolonien }\end{array}$ & 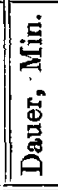 & 总 & 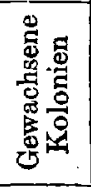 & & \\
\hline 1 & & & unzä & 10 & B. N & $\mathbf{u}$ & 5 & T. & 2460 & $87 \cdot 7$ & 48 \\
\hline 2 & l. & & unzäh & 10 & B.I & & $\approx$ & $\mathrm{T}$. & 4200 & & 20 \\
\hline 3 & 12. X. 16. & P. & , & 10 & B.N. & 29 & 5 & T. & 5100 & 74 & 48 \\
\hline 4 & 1 & P. & & 10 & B.N. & 16 & 5 & T. & 142 & $99 \cdot 29$ & 48 \\
\hline- & 16 & I. & 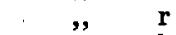 & 10 & B.N. & unzä & 5 & T. & 32 & & 48 \\
\hline 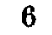 & 18 & . I. & (2) & 10 & B.N. & unz & 5 & T. & 70 & & \\
\hline 7 & 12 & . L. & , & 10 & B.N. & mehr als 10000 & 5 & $\mathrm{~T}$. & 23 & $99 \cdot 88$ & 48 \\
\hline 8 & 12 & & & 10 & B.N. & 4320 & 5 & $T$ & 138 & & 48 \\
\hline & & K. & ", & 10 & B. & 36 & 5 & T. & 125 & & 48 \\
\hline 10 & $12 . X$ & Schw. K. & ", & 10 & B.N & 1240 & $\mathbf{5}$ & & 100 & $99 \cdot 5$ & 48 \\
\hline
\end{tabular}

$r=$ rechte Hand

$1=$ linke Hand.

Aus der Tabelle ist ersichtlich, daß die Anzahl der Kolonien, die von den Händen vor der Desinfektion, den sogenannten Tageshänden, gewonnen waren, durchweg mehr als 20000 betrug. Diese hohe Zahl überimpfter Keime beweist im übrigen die recht intensive Art und Weise der angewandten Abimpfungsmethode. Während die zweite Hauptreihe zeigt, daß nach der Seifenwaschung eine -z. T. nur sehr geringe Keimabnahme, z. T. überhaupt keine zählbare Differenz in der Keimzahl auftrat, so ist aus der letzten Zahlenreihe, die die Ergebnisse der Alkoholwaschung und der beendeten Desinfektion enthält, doch größtenteils eine ganz bedeutende Keimabnahme zu konstatieren. So war in Fall 4, 5, 6, 7 und 8 der Keimgehalt der vollständig desinfizierten Hand um durchschnittlich 99.6 Proz. gegen den der Tageshand zurückgegangen, wobei zu berücksichtigen ist, daB die Zahl der von letzterer überimpften Keime nur mit 20000 in Rechnung gestellt ist, obgleich sie sicher in einzelnen Fällen bedeutend höhere Werte erreichte. Erwähnt sei noch, daß die Handoberfläche und ibre Bakterien- 
flora mit der Agarplatte und den auf ihr gewachsenen Kolonien nicht identifiziert werden kann, sondern daß man nur annähernde Werte bekommt. Denn bei dieser wie noch in höherem Maße bei den anderen Abimpfungsmethoden bleiben auf der Hand selbstverständlich Keime zurück, ein Umstand, der zwar bei der absoluten Keimzahlbestimmung eine Fehlerquelle darstellt, der aber bei diesen Untersuchungen, bei denen mit vergleichenden Zahlenwerten gerechnet wird, nicht weiter ins Gewicht fällt.

Die Resultate von Fall 1 bis 3 sind als schlecht zu bezeichnen, da die Keimabnahme im Vergleich zur Tageshand z. T. erheblich unter 90 Proz. beträgt. Dies ist um so auffälliger in Fall 3, der sich auf die rechte Hand der gleichen Versuchsperson bezieht, die linkerseits (Fall 4) ein verhältnismäßig gutes Resultat erzielte. Offenbar ist hier die rechte Hand nicht mit der gleichen Intensität bearbeitet worden wie die linke, was in der Rechtshändigkeit und in der Natur des Desinfektionsaktes begründet sein kann. Völlig unklar bleiben jedoch Fall 1 und 2; hier kann nur ein größerer Fehler mitspielen. Diese beiden Fälle glaube ich bei der Beurteilung der Wirksamkeit der Ahlfeld schen Methode ausschließen zu können, da die gefundenen Endwerte um so vielfaches alle übrigen übertreffen, daß bei einem derartigen Unterschied sicherlich nicht die Desinfektionsmethode als vielmehr andere äußere Umstände verantwortlich gemacht werden können. Sieht man also vón Fall 1 bis 3 mit ihren nicht in dem Desinfektionsmodus begründeten sçhlechten Resultaten ab, so muß man sagen, dảß die allgemein geübte Händedesinfektion mit einer Keimabnahme von rund 99.6 Proz. recht Gutes leistet.

Wird nun dasselbe günstige Resultat mit der Gochtschen Desinfekttionsmethode erreicht?

Die Gipswaschung, die die Seifenwaschung vertreten soll, besitzt ebenso wenig wie die letztere keimtötende Eigenschaften. Bei der Ahlfeldschen wie bei der Gochtschen Händedesinfektion ist das eigentlich Desinfizienz der Alkohol. Also liegt die Frage nahe, ob nicht eine der Alkoholwaschung vorhergehende gründliche Händereinigung überhaupt überflüssig ist. Und diese Frage ist auch aufgeworfen worden, und gerade in neuerer Zeit. Zahlreiche Autoren (v. Brunn, Heck u. a.) bejahen diese Frage nicht nur, sondern stellen sich sogar auf den Standpunkt, daB ein gründliches Abseifen oder sonst irgend ein mechanisches Bearbeiten. der Hände vor der Alkoholdesinfektion aus dem Grunde zu verwerfen sei, weil dadurch die Keime, die in den Schlupfwinkel und Poren der Hand versteckt sitzen, mobilisiert und an die Oberfläche hervorgeholt würden. Im Gegenteil solle man diese tiefsitzenden Bakterien ruhig in ihren Verstecken lassen und sie vielmehr dort durch Alkohol und ähnliche Mittel fixieren. Dieser Ansicht 
wäre zweifelsohne nicht zu widersprechen, wenn wir die Sicherheit hätten, daß die so in der Tiefe festgelegten. Keime tatsächlich auch während des Verlaufes der ganzen Operation fixiert blieben. Ich kann mich dem Einwande jedoch nicht verschließen, daß bei länger dauernden operativen Eingriffen, besonders bei solchen der Peritonealhöhle, die Hände des Operateurs so durch Blut, Gewebsflüssigkeiten usw. aufgeweicht werden, dab die fixierende Wirkung des Alkohols aufgehoben werden muß, abgesehen davon, daß eine leicht eintretende Schweißsekretion die Bakterien aus der Tiefe herausbefördert. Dies gilt vor allem für Operieren ohne Handschuhe oder mit solchen aus Zwirn; aber auch die Gummihandschuhe bieten bei ihrer leichten Verletzlichkeit keine sichere Gewähr für völligen Abschluß der Hand gegen die Operationsstelle. Es pflegt die Hand unter der Gummibekleidung recht stark zu transpirieren, so daß bei einem Undichtwerden des Handschuhes die Infektionsgefahr nicht gerade gering zu veranschlagen ist. Von diesem Gesichtspunkte aus halte ich eine der Alkoholwaschung vorausgehende recht eingehende mechanische Reinigung der Hände für dringend geboten; denn je mehr Keime an die Oberfläche befördert und abgetötet werden, um so weniger bleiben in der Tiefe, die später unter Umständen gefährliche Infektionen der Wundstelle hervorrufen können. Auf die Versuche, die diese Ansicht beweisen sollen, wird weiter unten noch eingegangen werden. Den Einwand, daß der Alkohol weniger bakterizide als vielmehr hautgerbende adstringierende und keimfixierende Eigenschaften besitze, hat bereits Ahlfeld durch eingehende Versuche widerlegt, zu welchem Resultate auch Fürbringer gelangt ist. Auch meine soeben erwähnten Versuche beweisen die bakterientötende Eigenschaft des Alkohols. In einer intensiven mechanischen Händereinigung müssen wir also unbedingt einen wesentlichen Faktor der ganzen Desinfektion erblicken, aber nur eben einen Bestandteil, denn nach dem Vorangegangenen ist es klar, daß auf die rein mechanische Waschung noch eine solche mit einer bakteriziden Lösung zu folgen hat. Deswegen mußte auch eine Desinfektion ausschließlich mit der Marmorstaubseife Schleichs und der Sängerschen Handseife die schlechten Resultate zeitigen, zu denen Paul und Sarwey in ihren Experimentaluntersuchungen über Händedesinfektion gelangten. Also gründliche, mechanische Aufweichung und Auflockerung der Haut und eine sich daran anschließende Waschung mit einem keimtötenden Mittel, das sind die beiden Faktoren, die allein eine wirklich gründliche Händedesinfektion garantieren, und aus denen sich auch die Ahlfeldsche Desinfektion zusammensetzt. Auch bei der Gips-Alkohol-Methode Gochts wird diesen grundsätzlichen Forderungen Rechnung getragen. Hierbei muß darauf hingewiesen werden, daß das Gipspulver, so wie es der Tonne entnommen wird, steril 
ist. Dies haben meine mehrfach wiederholten Versuche mit Agar-Nährböden und Traubenzucker-Hochagar ergeben.

Die Versuchsresultate dieser Desinfektionsmethode sind in Tabelle 2 zusammengestellt. Zum Vergleich mit den Ergebnissen der Ahlfeldschen Methode können sie deshalb herangezogen werden, weil sie unter genau denselben Bedingungen gewonnen sind wie jene, nur daß statt der Seifenwaschung eine solche mit feinstem Alabastergipspulver vorgenommen wurde in der Art und Weise, wie es Gocht in seiner erwähnten Veröffentlichung beschreibt.

Tabelle 2.

\begin{tabular}{|c|c|c|c|c|c|c|c|c|c|c|c|}
\hline \multirow[b]{2}{*}{ Nr. } & \multirow[b]{2}{*}{ Datum } & \multirow[b]{2}{*}{ Person } & \multirow{2}{*}{$\begin{array}{c}\text { Kolonien } \\
\text { von der } \\
\text { Tageshand } \\
\text { vor der } \\
\text { Desinfektion }\end{array}$} & \multicolumn{3}{|c|}{ Gipswaschung } & \multicolumn{3}{|c|}{$\begin{array}{c}70 \% \text { Alkohol } \\
\text { waschung }\end{array}$} & \multirow{2}{*}{ 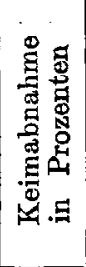 } & \multirow{2}{*}{ 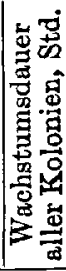 } \\
\hline & & & & 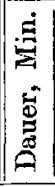 & 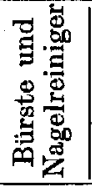 & $\begin{array}{c}\text { Gewachsene } \\
\text { Kolonien }\end{array}$ & $\begin{array}{l}j \\
\vdots \\
0 \\
0\end{array}$ & 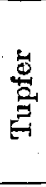 & 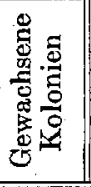 & & \\
\hline 1 & & $\mathbf{H}$ & unzählige $\mathrm{r}$ & 10 & B. & un & 5 & $\mathrm{~T}$. & 40 & 80 & 48 \\
\hline 2 & & & & 10 & B. I & & 5 & T. & 2 & & 4 \\
\hline 3 & 18. XII. 16. & $\mathrm{St}$ & & 10 & B. N. & unz & 5 & $\mathrm{~T}$. & 4 & 76 & 48 \\
\hline 4 & 18.XII. 16. & St. & & 10 & B. N. & unzähli & 5 & T. & 33 & & 48 \\
\hline $\mathbf{5}$ & & St. & $10000 \mathrm{r}$ & 10 & B. N. & über 10 & 5 & $\mathrm{~T}$ & 12 & & 48 \\
\hline 政 & {$[17$} & St. & 60001 & 10 & B. N. & über 1000 & 5 & T. & 8 & & \\
\hline 7 & 23 & St. & $5000 \mathrm{r}$ & 10 & B. N. & 200 & 5 & T. & 3 & & 48 \\
\hline 8 & 23. I. 17 & St. & 60001 & 10 & B. $\mathrm{N}$. & 600 & 5 & T. & 8 & $99 \cdot 87$ & 48 \\
\hline
\end{tabular}

$\mathrm{r}=$ rechte Hand, 1 = linke Hand.

Auch aus dieser Zusammenstellung ist ersichtlich, daß die Keimzahl nach der vorbereitenden Gipswaschung fast kaum abgenommen hat. Meist war noch eine Vermehrung der an und für sich schon unzählbaren Keimzahl der Tageshand zu konstatieren. Wie die dritte Hauptreihe zeigt, ist auch hier, und zwar in jedem Falle die Anzahl der nach beendeter Gesamtdesinfektion gewachsenen Kolonien im Vergleich zu der Menge, die vor der Desinfektion an der Handoberfläche haftete, ganz erheblich zurückgegangen. Im günstigsten Falle beträgt die Keimabnahme 99.94 Proz., durchschnittlich über $99 \cdot 85$ Proz. Wenn man demgegenüber stellt, daß der Durchschnitt der Keimverminderung bei der üblichen Desinfektionsmethode 99.6 Proz. (vgl. Tab. 1) betrug, so müßte man, schon rein äußerlich diese Zahlenwerte beurteilend, der Gochtschen Methode die bessere Wirkungsweise zuerkennen. Wenn auch vor einem festlegenden Urteil über die Güte der Methode die Zahl der Versuche erst noch bedeutend erweitert werden müßte, so läßt sich doch sehon aus meinen Versuchen schließen, daß die Gips-Alkohol- 
Desinfektion zum mindesten nich: schlechter ist als die gewöhnlich geübte Methode. Wenn ich soeben schrieb "schon rein äußerlich", so wollte ich damit das betonen, daß eine Waschung mit Gips viel intensiver ouf die Haut einwirkt als eine solche mit Seife, dringt der Gips doch vermöge seiner Form und Konsistenz in die feinsten Poren und Fälichen der Haut und reißt beim Herauswaschen und Bürsten die tiefsitzenden Bakterien mit oder macht den Weg frei, auf dem der Alkohol zu ihnen geləngen und sie abtöten kann. Bei genügendem Bürsten und Abspülen bleibt nichts vom Gipspulver auf und in der Haut zurück, wovon man sich durch Betrachten der Hand und Fingernägel überzeugen kann, auch habe ich nie ein Gipskörnchen in dem beimpften Agar finden können. Dabei verursacht ein Waschen mit dem Pulver nicht das geringste unangenehme Gefühl an der Hand, im Gegenteil hat der Gips den Vorzug, nicht soviel Hautfett zu lösen wie Seife, die vermöge ihrer Eigenschaft leicht die Haut rissig macht. Risse und Rhagaden der Hände erschweren an und für sich schon eine gründliche Händedesinfektion.

Nachdem sich also die Gips-Alkoholmethode als brauchbar erwiesen zu haben schien, lag mir daran, in Erfahrung zu bringen, ob nicht auch andere pulverisierte Substanzen dieselbe hautreinigende Eigenschaft zeigen. Einige dieser kursorischen Versuche sind in Tabelle 3 zusammengestellt. Die Versuchsanordnung blieb die gleiche, nur trocknete ich mir hierbei wie auch bei allen übrigen folgenden Versuchen nach der Alkoholwaschung nicht mehr die Hände mit einem sterilen Tupfer ab, sondern ließ sie, wie das ja auch in praxi geschieht, lufttrocken werden. Wie Tabelle 3 zeigt, versuchte ich zunächst das Kalziumkarbonat. Das Ergebnis war auch bei wiederholten Versuchen kein zufriedenstellendes, da die Keimzahl nach beendeter Desinfektion immer noch $1 / 2$ bis $1 / 10$ der vor derselben ermittelten Anzahl betrug. Als brauchbarer zur Vorbehandlung erwies sich die Tonseife, wie sie jetzt im Handel ist. Aus praktischen Gesichtspunkten dürfte sie zur allgemeinen Benutzung ungeeignet erscheinen da sie sich in den Leitungsröhren niederschlägt und eine Verstopfung derselben hervorruft; in Berlin sollen bei dem jetzigen starken Gebrauche der Tonseifen selbst die größten Kanalisationsrohre allmählich verstopft worden sein. Bei Gips ist dies nicht der Fall. Selbst hartgewordene Gipsmassen lassen sich durch salzhaltiges Wasser, wie es ja immer in größeren Rohren vorhanden ist, wieder zur Auflösung bringen. In den engen Abflußrohren, die unmittelbar an die Wascheinrichtung angeschlossen sind, wird im allgemeinen ein Hartwerden des Gipses nicht in Betracht kommen, da die Gipswaschung im strömenden Wasser erfolgt und mit einem beträchtlichen Wasserüberschuß beim Waschen zu rechnen ist, der eine Erhärtung solange hintanhält, bis das Gipswasser das System enger Röhren verlassen hat. 
Tab elle

\begin{tabular}{|c|c|c|c|c|c|c|}
\hline Nr. & Datum & Person & $\begin{array}{l}\text { Kolonien von } \\
\text { der Tageshand } \\
\text { vor der } \\
\text { Desinfektion }\end{array}$ & Art des Mittels & $\begin{array}{c}\text { Dauer } \\
\text { Min. }\end{array}$ & $\begin{array}{c}\text { Bürste? } \\
\text { Nagel- } \\
\text { reiniger? } \\
\text { flie日endes } \\
\text { Wasser? } \\
\end{array}$ \\
\hline 1 & 2. X. 16. & Dr. Sch. & unzählige $\mathbf{r}$ & Calc. carbonic. & 10 & B. \\
\hline 2 & $2 . \mathrm{X} .16$ & Dr. Sch. & unzählige 1 & Calc. carbonic. & 10 & B. \\
\hline $\mathbf{l} \mathbf{a}$ & 9. XI. 16 . & & unzählige $\mathbf{r}$ & Calc. carbonic. & 10 & B.N. \\
\hline $2 a$ & 9. XI. 16. & St. & $6000 \mathrm{l}$ & Calc: carbonic. & 10 & B.N. \\
\hline 3 & 5. X. 16. & Frl. G. & $350 \mathrm{r}$ & Tonseife & 10 & - \\
\hline 4 & 5. X. 16. & Frl. G. & unzählige 1 & Tonseife & 10 & - \\
\hline 5 & 16. X. 16. & St. & unzählige $r$ & fein pulv. Schmirgel & 10 & B. N. \\
\hline 6 & 16. X. 16. & St. & 5201 & fein pulv. Schmirgel & 10 & B. N. \\
\hline 7 & 20. X. 16 . & St. & $103 \mathrm{r}$ & fein pulv. Bimstein & 10 & B. N. \\
\hline 8 & $20 . X .16$. & St. & 25001 & fein pulv. Bimstein & 10 & B. N. \\
\hline 9 & 26. X. 16. & St. & unzählige $\mathbf{r}$ & $\begin{array}{c}\text { Gips-Soda-Brei } \\
\text { (250 ccm Soda } 5 \text { Proz. }\end{array}$ & 10 & B. N. \\
\hline 10 & $26 . \times 16$ & St. & $3500 \mathrm{l}$ & $\begin{array}{l}\text { Gips } 100 \text { g) } \\
\text { Gips-Soda-Brei } \\
\text { (wie Nr. 9) }\end{array}$ & 10 & B. N. \\
\hline 11 & 9. XI. 16. & St. & $360 r$ & $\begin{array}{c}\text { Gips-Soda-Brei } \\
(250 \mathrm{ccm} \text { Soda } 2 \text { Proz. } \\
\text { Gips } 100 \mathrm{~g})\end{array}$ & 10 & B. N. \\
\hline 12 & 9. XI. 16. & St. & 90001 & $\begin{array}{l}\text { Gips-Soda-Brei } \\
\text { (wie Nr. 11) }\end{array}$ & 10 & B. N. \\
\hline
\end{tabular}

Aus dem dargelegten Grunde dürfte demnach praktiseherseits eine T'onseife zur Händedesinfektion nicht in Betracht kommen, ebensowenig wie fein gemahlener Schmirgel. Wenn auch ein Versuch mit dieser Substanz theoretisch ein recht annehmbares Resultat zeitigte (vgl. Fall 5 u. 6), so ist ihre Wirkungsweise auf die Haut infolge ihrer Beschaffenheit viel zu intensiv, als $d a ß$ sie praktisch wiederholt Anwendung finden könnte, ohne hochgradige Schädigungen und Rhagadenbildungen der Epidermis zu erzeugen: Der sich daran anschließende Versuch mit pulverisiertem Bimstein entsprach, wie Fall 8 zeigt, nicht den Erwartungen.

Weiter prüfte ich ein Gips-Soda-Gemisch. $250 \mathrm{ccm}$ einer $2 \mathrm{bzw} .5 \mathrm{pro-}$ zentigen sterilen Sodalösung wurden in dünnem Strahle $100 \mathrm{~g}$ Gipspulver zugesetzt; so daß schlieBlich ein Brei von mittlerer Konsistenz entstand, mit dem ich mich dann so wie mit Schmierseife wusch. In Fall 9 und 10 benutzte ich eine 5prozentige, in Fall 11 und 12 eine 2 prozentige Sodalösung. Mit dem 5 prozentigen Soda-Gipsbrei wurde ein recht zufriedenstellendes Desinfektionsergebnis erzielt (in Fall 9 beträgt die Keimabnahme $99 \cdot 87$ Proz.), wogegen sich das 2 prozentige Sodagemisch als völlig desinfektionsuntüchtig herausstellte. Aber auch von einer Waschung mit einem 5prozentigen 
3.

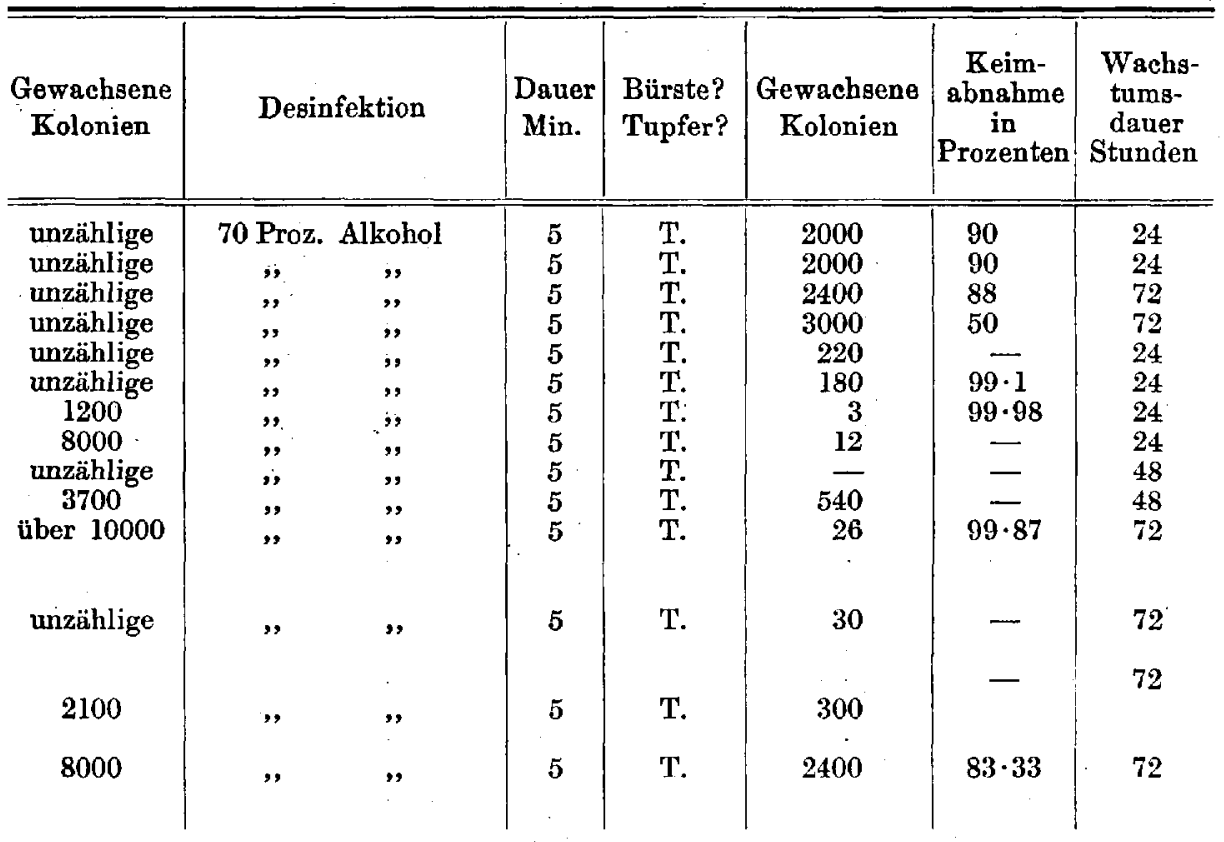

Gips-Sodabrei muB in der Praxis Abstand genommen werden, da der Brei nach ungefähr 10 Minuten erstarrt und damit unbrauchbar wird. Er müßte daher jedesmal vor der Desinfektion frisch zubereitet werden. Von allen diesen mechanischen Reinigungsmitteln dürfte also keines zur Vorbehandlung der Hände brauchbar sein, sei es nun, daß die Intensität ihrer Wirkungsweise zu gering ist, wie z.B. bei dem Kalziumkarbonat, sei es, daß diese andrerseits zu stark ist, z. B. beim Schmirgel, oder mögen ihrer Anwendbarkeit trotz ihrer guten Wirkung sonstige technische und praktische Bedenken und Fehler im Wege stehen. Dabei sei im voraus auf die Untersuchungen über das Baryumsulfat hingewiesen, über die ich aus rein äuBeren Gründen erst am Schlusse der Arbeit berichten werde.

Im folgenden habe ich die Frage bearbeitet, welche Konzentration des Alkohols bei dem Gipsverfahren die besten Ergebnisse zeitigt.

Steigt die Desinfektionskraft des Alkohols mit seinen höheren Konzentrationen? Epstein gelang es, den Nachweis zu erbringen, „daß der absolute Alkohol gegenüber angetrockneten vegetativen Keimen absolut wirkungslos ist", was auch andere Untersucher bestätigt haben. Der Grund hierfür liegt bekanntlich darin; daß durch die eiweißfällende und schrumpfende 
Tabelle

\begin{tabular}{|c|c|c|c|c|c|c|c|}
\hline \multirow[b]{2}{*}{ Nr. } & \multirow[b]{2}{*}{ Datum } & \multirow[b]{2}{*}{ Person } & \multirow[b]{2}{*}{$\begin{array}{c}\text { Kolonien } \\
\text { von der } \\
\text { Tageshand } \\
\text { vorder } \\
\text { Desinfektion }\end{array}$} & \multicolumn{3}{|c|}{ Gips-Waschung } & \multirow[b]{2}{*}{ Desinfiziens } \\
\hline & & & & 离 & 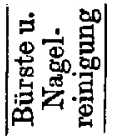 & $\begin{array}{c}\text { Gewachsene } \\
\text { Kolonien }\end{array}$ & \\
\hline 1 & 31. X. 16. & St. & $2400 \mathrm{r}$ & 10 & B. N. & 2800 & Seifenspir. \\
\hline 2 & 31. X. 16. & St. & 28001 & 10 & B. N. & unzählige & Seifenspir. \\
\hline 3 & 2. XI. 16. & St. & über $10000 \mathrm{r}$ & 10 & B. N. & 1200 & 50pr. Alk. \\
\hline 4 & 2. XI. 16. & St. & über $10000 \mathrm{I}$ & 10 & B. N. & unzählige & 50pr. Alk. \\
\hline 5 & 6. XI. 16. & St. & unzählige $\mathbf{r}$ & 10 & B. N. & unzählige & 60pr. Alk. \\
\hline 6 & 6. XI. I6. & St. & unzählige l & 10 & B. N. & unzählige & $60 \mathrm{pr}$. Alk. \\
\hline 7 & 8. XI. 16. & St. & unzählige $\mathbf{r}$ & 10 & B. N. & unzählige & 60 pr. Alk. \\
\hline 8 & 8. XI. 16. & St. & unzählige I & 10 & B. N. & unzählige & 60pr. Alk. \\
\hline 9 & 22. I. 17. & St. & über $10000 \mathrm{r}$ & 10 & B. N. & 800 & $60 \mathrm{pr}$. Alk. \\
\hline 10 & 22. I. 17. & St. & über 10000 l & 10 & B. N. & 2500 & 60pr. Alk. \\
\hline 11 & 12. X. 16 & Schw. I. & unzählige r & 10 & B. N. & unzählige & 70pr. Alk. \\
\hline 12 & $12 . \times .16$. & Sohw. I. & unzählige l & 10 & B. N. & 3600 & 70pr. Alk. \\
\hline 13 & 18. XII. 16. & St. & unzählige $r$ & 10 & B. N. & unzählige & $70 \mathrm{pr}$. Alk. \\
\hline 14 & 18. XII. 16. & St. & unzählige I & 10 & B. N. & unzählige & $70 \mathrm{pr}$. Alk. \\
\hline 15 & 16. I. 17. & St. & $10000^{\circ} \mathrm{r}$ & 10 & B. N. & über 10000 & 70pr. Alk. \\
\hline 16 & 16. I. 17. & St. & $6000 \mathrm{I}$ & 10 & B. $\mathbf{N}$. & über 10000 & 70pr. Alk. \\
\hline 17 & 23. I. 17. & St. & $5000 \mathrm{r}$ & 10 & B. N. & 200 & $70 \mathrm{pr}$. Alk. \\
\hline 18 & 23. I. 17. & St. & $6000 \mathrm{l}$ & 10 & B. N. & 600 & 70pr. Alk. \\
\hline 19 & $20 . \mathrm{XI} .16$. & St. & unzählige $\mathrm{r}$ & 10 & B. N. & unzählige & 80pr. Alk. \\
\hline 20 & 20. XI. 16. & St. & unzählige l & 10 & B. N. & unzählige & 80 pr. Alk. \\
\hline 21 & $20 . \mathrm{XI} .16$. & St. & unzählige $r$ & 10 & B. N. & unzählige & $80 \mathrm{pr}$. Alk. \\
\hline 22 & 20. XI. 16 . & St. & unzählige I & 10 & B. N. & unzählige & 80pr. Alk. \\
\hline 23 & 21. XI. 16. & St. & $3000 \mathrm{r}$ & 10 & B. N. & 2500 & abs. Alk. \\
\hline 24 & 21. XI. 16. & St. & unzählige I & 10 & B. N. & unzählige & abs. Alk. \\
\hline 25 & 11. XI..16. & Prof. G. & $600 \mathrm{r}$ & 10 & B. - - & 700 & Alk.-Par. \\
\hline 26 & 11. XI. 16. & Prof. G. & $1200 \mathrm{l}$ & 10 & B. - & 1500 & Alk.-Par. \\
\hline
\end{tabular}

Wirkung von Alkoholen höherer Konzentration sein Vordringen in das Innere der Bakterien unmöglich gemacht wird. Weigls Schüttelversuche haben dies bewiesen. Allerdings genügt in manchen Fällen die wasserentziehende und austrocknende Wirkung des abșoluten Alkohols zur Abtötung der Keime, jedoch versagt diese vollkommen, wenn es sich um Vernichtung von Sporen handelt. Der grundsätzliche Unterschied zwischen der Wirkung des absoluten Alkohols und des mit Wasser verdünnten besteht darin, dab der erstere nur durch Austrocknung keimtötend wirkt, während letzterem eine wirklich desinfizierende Kraft innewohnt, und bei ihm eine Diffusion des Alkohols in dem durch das Wasser gequollenen Bakterienleib ermöglicht wird. Während also auf angetrocknete vegetative Bakterien schwächer konzentrierte Alkohole besser einwirken, so werden Bakterien in feuchtem, wasserhaltigem Zustande auch von absolutem Alkohol in gleichem Maße vernichtet, woil dieser durch den Wassergehalt der Keime selbst eine Verdünnung erfährt und es sich dann nicht mehr um absoluten Alkohol handelt 
4.

\begin{tabular}{|c|c|c|c|c|c|}
\hline $\begin{array}{l}\text { Dauer } \\
\text { Min. }\end{array}$ & $\begin{array}{l}\text { Bürste? } \\
\text { Tupfer ? }\end{array}$ & $\begin{array}{c}\text { Gewachsene } \\
\text { Kolonien }\end{array}$ & $\begin{array}{c}\text { Keim- } \\
\text { abnahme } \\
\text { in Prozenten }\end{array}$ & \begin{tabular}{|c|} 
Wachstums- \\
dauer \\
allerKolonien \\
Std.
\end{tabular} & Bemerkungen. \\
\hline $\begin{array}{l}5 \\
5 \\
5 \\
5 \\
5 \\
5 \\
5 \\
5 \\
5 \\
5 \\
5 \\
5 \\
5 \\
5 \\
5 \\
5 \\
5 \\
5 \\
5 \\
5 \\
5 \\
5 \\
5 \\
5 \\
5 \\
5\end{array}$ & 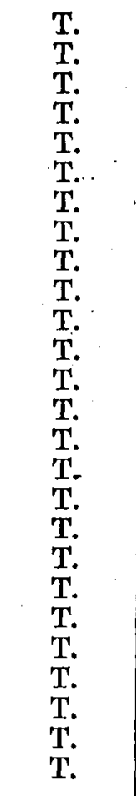 & $\begin{array}{r}55 \\
100 \\
600 \\
2100 \\
\mathbf{1 3} \\
\mathbf{2 5 0} \\
\mathbf{8} \\
\mathbf{3 6} \\
\mathbf{1 2} \\
\mathbf{8} \\
\mathbf{4 0} \\
\mathbf{2 3} \\
\mathbf{4 8} \\
\mathbf{3 3} \\
\mathbf{1 2} \\
\mathbf{8} \\
\mathbf{3} \\
\mathbf{8} \\
2 \\
\mathbf{7 0} \\
\mathbf{2} \\
70 \\
20 \\
63 \\
108 \\
80\end{array}$ & $\begin{array}{l}97 \cdot 71 \\
96 \cdot 43 \\
94 \cdot 0 \\
79 \cdot 0 \\
99 \cdot 93 \\
98 \cdot 75 \\
99 \cdot 96 \\
99 \cdot 82 \\
99 \cdot 88 \\
99 \cdot 92 \\
99 \cdot 8 \\
99 \cdot 88 \\
99 \cdot 76 \\
99 \cdot 83 \\
99 \cdot 88 \\
99 \cdot 87 \\
99 \cdot 94 \\
99 \cdot 87 \\
99 \cdot 99 \\
99 \cdot 65 \\
99 \cdot 99 \\
99 \cdot 65 \\
99 \cdot 33 \\
99 \cdot 68 \\
82 \cdot 0 \\
93 \cdot 33\end{array}$ & $\begin{array}{l}48 \\
48 \\
72 \\
72 \\
24 \\
24 \\
24 \\
24 \\
48 \\
48 \\
48 \\
48 \\
48 \\
48 \\
48 \\
48 \\
48 \\
48 \\
24 \\
24 \\
24 \\
24 \\
48 \\
48 \\
48 \\
48\end{array}$ & $\begin{array}{l}\text { s. Tabelle } 6 . \\
\text { s. Tabelle } 6 . \\
\text { s. Tabelle 1. } \\
\text { s. Tabelle } 1 . \\
\text { s. Tabelle } 1 . \\
\text { s. Tabelle 1. } \\
\text { s. Tabelle 1. } \\
\text { s. Tabelle 1. } \\
\text { s. Tabelle I u. } 6 . \\
\text { s. Tabelle I u. } 6 . \\
\text { s. Tabelle 6. } \\
\text { s. Tabelle } 6 . \\
\text { s. Tabelle 6. } \\
\text { s. Tabelle 6. } \\
\text { s. Tabelle 6. } \\
\text { s. Tabelle 6, } \\
\text { 7 Std. vorher wegen Ope- } \\
\text { ration desinfiziert. }\end{array}$ \\
\hline
\end{tabular}

(Winkler, Hansen). Epstein, Minervini u.a. schreiben dem Äthylalkohol in 50 bis 70 prozentiger Lösung die beste Wirkungsweise zu.

Aus Tabelle 4 sind meine diesbezüglichen. Untersuchungsergebnisse zu ersehen. Versuch 1 und 2, nur der Vollständigkeit halber angestellt, zeigen, daß der Seifenspiritus nach Mikulicz ein gutes Resultat zu ergeben scheint. Es muß erwähnt werden, daß dieser Versuch an einem Tage gemacht wurde, an dem die Hände schon mehrfach starken Waschungen ausgesetzt waren, so da $B$ der Keimgehalt der Hände vor Beginn des Versuchs schon ein geringer war. Auch hier zeigte es sich wieder, daß die linke Hand, die von der rechten Hand viel energiseher gewaschen wird, mehr Keime nach der Waschung mit Gips aufweist als die rechte. Das Schlußresultat nach 5 Minuten langer Waschung mit Seifenspiritus zeigt eine Keimabnahme der rechten Hand von $97 \cdot 7$ Proz., der linken Hand von $96 \cdot 43$ Proz.

Versuch 3 und 4, Gipswaschung mit nachfolgender 50 prozentiger Alkoholwaschung, ergab eine Keimabnahme der rechten Hand um 94 Proz., 
der linken Hand um 79 Proz., den Ansprüchen an eine ausreichende Desinfektionswirkung nicht entsprechende Resultate.

Ganz anders verhält es sich dagegen.bei Anwendung von 60 prozentigem Alkohol. Hier beträgt die durchschnittliche Keimabnahme über 99.6 Proz., in drei Viertel der Fälle aber über $99 \cdot 9$ Proz. Vergleichsweise sind hier noch einmal die Desinfektionsresultate mit 70prozentigem Alkohol aufgefïhrt, die eine Keimabnahme von über 99.8 Proz. ergaben; eine gleich große Keimverminderung wird mit 80prozentigem Alkohol erreicht, wie aus Fall 19 und 20 ersichtlich. Nicht ganz so gut aber immerhin noch recht annehmbar ist das bei Benutzung von absolutem Alkohol gewonnene Resultat (Fall 23 und 24). Was die Wirkungsweise eines Gemisches von 70 Proz. Alkohol und Paraffin $z u$ gleichen Teilen anbelangt, so lieB sich eine Keimabnahme von 82 Proz. bzw:" 93.3 Proz. feststellen (Versuch 25 und 26). Wenn auch das Ergebnis dieses Paraffin-Alkohol-Gemisches prozentualiter hinter den anderen in Tabelle 4 aufgezählten Ergebnissen zurücksteht, so hat die Anwendung dièses Gemisches den Vorzug für sich, da $\mathfrak{B}$ eine größtmögliche Schonung der Haut damit verbunden ist. Zieht man die Schlußfolgerung aus den Untersuchungsergebnissen dieser Versuchsreihe, so muß man sagen, daß ein bedeutender Unterschied in der keimabtötenden Wirkung der Alkohole in den Konzentrationsstufen von 60 Proz. aufwärts bis zum absoluten Alkohol so gut wie nicht zu erkennen ist; das wäre im allgemeinen das gleiche Resultat, zu dem schon Winkler u. a. gelangten. Aber noch ein zweites beweist diese Versuchsgruppe mit ihren z. T. ausgezeichneten Desinfektionserfolgen, nämlich die gute Wirkung der Vorbehandlung der Hände durch eine Waschung mit Gipspulver mit nachfolgender Waschung mit 60prozenttigem Alkohol, die sich in mehreren Versuchen wiederholentlich bestätigte.

Bei der Beurteilung der folgenden in Tabelle 5 zusammengestellten Untersuchungen muß man meine früheren Bemerkungen über hygienische und chirurgische Händedesinfektion berïcksichtigen, nach denen diese Versuche (mit vorangehender künstlicher Infektion) nur ein rein theoretisches Interesse haben können. Sie bilden nur eine Ergänzung der oben geschilderten Untersuchungen über die desinfizierende Wirkung der Gochtschen Methode und zwar diesmal bestimmten Bakterien gegenüber, zum Teil recht resistenter Natur. Ich wählte den Bazillus prodigiosus, das Bakt. coli commune, die Sareina aurantiaca und den Staphylokokkus pyogenes albus bzw. aureus zur künstlichen Infektion der Hand. Im Gegensatz zu den Untersuchern, die sich mit der hygienisehen Desinfektion beschäftigten und daher die Händeinfektion in der Weise vornahmen, daß sie künstlich mit bestimmten Bakterien infizierte Wäschestücke anfaßten oder wie Börnstein mit Bouillonabschwemmungen von Agarkulturen getränktes Filtrier- 
papier unter den Nägeln und an den Endphalangen der Finger entlang strichen, also nur eine ganz oberflächliche Infektion vornahmen, ging ich in der Weise vor, daß ich mir ungefähr $8 \mathrm{ccm}$ einer 24stünd. Bouillonkultur oder Kochsalzaufschwemmung von bestimmten Schrägagarkulturen nach und nach in die Hohlhand gießen ließ und diese dann mit einem sterilen Tupfer intensiv auf die Handflächen und Rücken und unter die Fingernägel einrieb. Darauf ließ ich die Bakterien mindestens 5 Minuten lang eintrocknen. Ich hatte nicht die Absicht, eine am Krankenbett vorkommende Infektion künstlich nachzuahmen, sondern es sollte sich. zeigen, wieweit bestimmte tiefsitzende Bakterien durch die Gips-Alkohol-Methode beseitigt würden. Bei diesen Versuchen war es natürlich von Vorteil, wenn die Infektionen an möglichst keimarmen Händen erfolgten, so daß möglichst nur die für die Infektionen gewählten Bakterien zum Wachstum kommen konnten. Ich nahm deshalb vor der Infektion eine gründliche Desinfektion der Hände vor (eine der oben beschriebenen Methoden). Anschließend daran wurden die Hände, um noch die letzten Spuren. von Alkohol zu entfernen, mit heißem Wasser gründlich abgespült. Jetzt erst erfolgte die Infektion in der angegebenen Weise. Nach völligem Antrocknen der Bakterien wurde eine Abimpfung der Hände nach der von mir geübten Methode vorgenommen. Es folgte Gipswaschung, wiederum Abimpfung, Anwendung. von 70 Proz. Alkohol und nochmalige Abimpfung in der oben angegebenen Weise.

Bei dem Versuch mit dem Baz. prodigiosus wurden die beimpften Agarplatten einer Temperatur von $22^{\circ}$ ausgesetzt; bei den mit dem Baz. coli infizierten Händen erfolgte die Abimpfung mit flüssig gemachtem Endoagar. In den Versuchen 1 bis 4 wurde 70prozentiger, 5 bis 8 und 11 und 1260 prozentiger und 9 und 10 80prozentiger Alkohol verwendet.

Fall 1 und 2 der Tabelle 5 behandeln die Prodigiosus-Infektion. Es zeigt sich im Kulturversuch, daß auf der Handoberfläche überhaupt keine Keime lebensfähig geblieben sind; offenbar waren sie schon durch die $10 \mathrm{Mi}$ nuten lange Austrocknung zugrunde gegangen. Die durch die Gipswaschung aus der Tiefe hervorgeholten Keime wurden dann durch die anschließende Alkoholdesinfektion sämtlich abgetötet. Nur in Fall 2 gelangte eine Kolonie nach 72 Stunden zur Entwicklung. Dieses günstige Ergebnis erklärt sich durch die geringe Widerstandsfähigkeit des Bacillus prodigiosus. Auch bei der Coli-Infektion wurde unter 4 Fällen 3 mal völlige Sterilität erreicht, in dem vierten (Fall 4) entwickelten sich 2 Coli-Kolonien. Während in Fall 5 und 6, wie zu erwarten war, nach der Gipswaschung eine Zunahme der Keimmenge erfolgte, so nahm diese in Fall 3 und 4 nach der Gipsreinigung ganz bedeutend ab. Der Grund hierfür ist vielleicht darin zu suchen, daß die Bakterien nicht intensiv genug in die Epidermis eingerieben worden waren. 
Tabelle

\begin{tabular}{|c|c|c|c|c|c|c|c|c|}
\hline \multirow[t]{2}{*}{ Nr. } & \multirow[t]{2}{*}{ Datum } & \multirow[t]{2}{*}{ 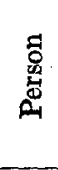 } & \multirow[t]{2}{*}{$\begin{array}{l}\text { Bakterien- } \\
\text { Auf- } \\
\text { sohwemmung }\end{array}$} & 息高 & 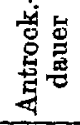 & \multirow[t]{2}{*}{$\begin{array}{c}\text { Gewachsene } \\
\text { Kolonien }\end{array}$} & $\stackrel{\mathscr{Q}}{\tilde{E}}$ & \multirow[t]{2}{*}{$\begin{array}{l}\text { Gips - } \\
\text { Bürste ? } \\
\text { Nagel- } \\
\text { reiniger? }\end{array}$} \\
\hline & & & & Min. & Min. & & Min. & \\
\hline 1 & 5. X. 16. & St. & B. prodigiosus $r$ & 3 & 10 & steril & 10 & - \\
\hline 2 & 5. X. 16. & St. & B. prodigiosus 1 & 3 & 10 & steril & 10 & - \\
\hline $\mathbf{3}$ & 9. X. 16. & St. & Baz. coli $\mathbf{r}$ & 3 & 5 & 35 & 10 & B. N. \\
\hline 4 & 9. X. 16 . & St. & Baz. coli 1 & 3 & 5 & 1060 & 10 & B. N. \\
\hline 5 & 15. XI. 16. & St. & Baz. coli $\mathbf{r}$ & 3 & 5 & 16 & 10 & B. N. \\
\hline 6 & 15. XI. 16. & St. & Baz. coli I & 3 & 5 & 26 & 10 & B. N. \\
\hline 7 & 14. XI. 16. & St. & Staph. albus $\mathbf{r}$ & 3 & 5 & über 10000 & 10 & B. N. \\
\hline 8 & 14. XI. 16. & St. & Staph. albus I & 3 & 5 & über 10000 & 10 & B. N. \\
\hline 9 & 21. XI. 16. & St. & Staph. aureus $\mathbf{r}$ & 3 & 3 & unzählige & 10 & B. N. \\
\hline 10 & 21. XI. 16. & St. & Staph. aureus 1 & 3 & 3 & über 10000 & 10 & B. N. \\
\hline 11 & 20. XI. 16. & St. & Sarcin. aurant. $r$ & 3 & 3 & unzählige & 10 & B. N. \\
\hline 12 & 20. XI. 16. & St. & Sarcin. aurant. I & $\mathbf{3}$ & $\mathbf{3}$ & unzählige & 10 & B. N. \\
\hline
\end{tabular}

Handelte es sich bei den eben erwähnten Bakterienarten um solche von nur geringer oder sogar minimaler Widerstandskraft, so zeichnen sich die beiden nun folgenden Mikroorganismen (Fall 7 bis 10) durch eine recht erhebliche Resistenz aus. So beträgt die Keimabnahme bei der. Infektion mit dem Staphylokokkus albus 98.5 und 97.0 Proz. und bei der mit dem Staphylokokkus aureus durchschnittlich 99. 77 Proz. Sterilität wurde in keinem Falle erreicht. Trotzdem miussen diese Desinfektionsergebnisse, speziell die dem Staphylokokkus aureus gegeniiber erzielten als sehr zufriedenstellend bezeichnet werden. Die benutzten Staphylokokken-Kulturen waren so virulent, daß sie eine Paronychie bzw. ein Panaritium bei dem Untersucher nach kurzer Zeit hervorriefen. Noch besser zeigte sich die Desinfektionsfähigkeit der Gips-Alkohol-Methode bei der künstlichen Infektion mit der Sarcina aurantiaca (Fall 11 und 12). Waren nach der Infektion an jeder Hand unzählige Keime zu konstatieren, so war die Zahl nach vollendeter Desinfektion nach Gocht beiderseits auf nur 8 nachweisbare Keime gesunken $=99.96$ Proz.

Was besagen nun diese Untersuchungen und deren Resultate? Zum wenigsten wird durch sie der Beweis erbracht, daß bestimmte an der Handoberfläche fest haftende $z$. T. recht resistente und virulente Bakterien durch die Gochtsche Desinfektionsmethode in sehr großer Menge beseitigt und vernichtet werden. Ferner waren die meisten der Bakterien, die der Alkoholeinwirkung ausgesetzt waren, nicht jene, die urspünglich an der Handoberfläche hafteten. Diese letzteren wurden naturgemäß zum weitaus- 
5.

\begin{tabular}{|c|c|c|c|c|c|c|c|}
\hline \multirow{2}{*}{$\begin{array}{c}\text { waschung. } \\
\text { Gewachsene } \\
\text { Kolonien }\end{array}$} & \multirow{2}{*}{ 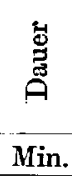 } & \multicolumn{3}{|c|}{ Alkoholwaschung } & \multirow{2}{*}{$\begin{array}{l}\text { Wachs- } \\
\text { tumskeim- } \\
\text { abnahme } \\
\text { in Proz. }\end{array}$} & \multirow{2}{*}{$\begin{array}{c}\text { Wachs- } \\
\text { tums- } \\
\text { dauer } \\
\text { aller Kol. } \\
\text { in Std. }\end{array}$} & \multirow[b]{2}{*}{$\begin{array}{c}\text { Be- } \\
\text { merkungen }\end{array}$} \\
\hline & & $\begin{array}{c}\text { Konzentr. } \\
\text { des } \\
\text { Alkohols } \\
\text { in Proz. }\end{array}$ & $\begin{array}{l}\text { Bürste? } \\
\text { Tupfer? }\end{array}$ & $\begin{array}{c}\text { Ge- } \\
\text { wachsene } \\
\text { Kolonien }\end{array}$ & & & \\
\hline 150 & 5 & 70 & $\mathrm{~T}$. & steril & - & 72 & \\
\hline 30 & 5 & 70 & $\mathrm{~T}$. & 1 & - & 72 & \\
\hline 1 & 5 & 70 & T. & steril & - & 24 & \\
\hline 50 & 5 & 70 & T. & 2 & - & 24 & \\
\hline 55 & 5 & 60 & T. & steril & - & 72 & \\
\hline 320 & 5 & 60 & T. & steril & - & 72 & \\
\hline 1200 & 5 & 60 & T. & 300 & 97 & 24 & \\
\hline 900 & 5 & 60 & T. & 150 & $98 \cdot 5$ & 24 & \\
\hline über 10000 & 5 & 80 & T. & 24 & $99 \cdot 88$ & 48 & s. Tabelle 7 . \\
\hline über 10000 & 5 & 80 & $\mathbf{T}$. & 34 & $99 \cdot 66$ & 48 & s. Tabelle 7 \\
\hline unzählige & 5 & 60 & T. & 8 & $99 \cdot 96$ & 24 & \\
\hline unzählige & 5 & 60 & 'T. & 8 & $99 \cdot 96$ & 24 & \\
\hline
\end{tabular}

größten Teile schon auf rein mechanische Weise durch die intensive Gipswaschung abgerieben und mit dem Leitungswasser fortgespült. Die größte Anzahl der durch den Alkohol vernichteten Keime stammte aus den tieferen Schichten der Haut und war dureh die Vorbehandlung mit Gips hervorgeholt oder sonst der Alkoholwirkung zugänglich gemacht worden. In neuerer Zeit ist die Ansicht vertreten, die in den Furchen und Vertiefungen sich aufhaltenden Bakterien $\mathrm{zu}$ belassen und sie dort zu fixieren. Es wird daher eine mechanische Vorbehandlung der Hände vor der Alkoholdesinfektion entweder ganz abgelehnt, oder nur eine ganz oberflächliche Seifenwaschung, die den gröbsten Handschmutz entfernen soll, befürwortet. Nach meinen Versuchen müßte man einer Händedesinfektion den Vorzug geben, die sich aus zwei Faktoren zusammensetzt, aus einer recht intensiven mechanischen Waschung der Hände bei möglichster Schonung der Haut und der darauf folgenden Alkoholdesinfektion, aus denen sich die Gips-HeißwasserAlkoholmethode zusammensetzt. Dab sich dies nicht nur theoretisch, wie oben, sondern auch durch Laboratoriumsversuche erhärten läßt, zeigen die Tabellen 6 und 7.

Ahlfelds Verfahren zum Nachweise, dàß Alkohol wirklich bakterizid wirke, bestand darin, daB er den desinfizierten Finger 2 Minuten lang in warmes, steriles Wasser hielt und dann durch nochmaliges Abimpfen zeigte, daß der vor der Wassereinwirkung möglichst keimfreie Finger auch nach dem Aufquellen der Epidermis und nach Aufhebung der fixierenden Wirkung des Alkohols keine Zunahme an Keimen aufwies. Daß die Hände bei vielen 
Tabelle 6 .

\begin{tabular}{|c|c|c|c|c|c|c|}
\hline \multirow{2}{*}{ Nr. } & \multirow{2}{*}{ Datum } & \multirow{2}{*}{ Person } & \multirow{2}{*}{$\begin{array}{l}\text { Kolonien von } \\
\text { der Tageshand }\end{array}$} & \multicolumn{3}{|c|}{ Gips-W Waschung } \\
\hline & & & & $\begin{array}{l}\text { Dauer } \\
\text { Min. }\end{array}$ & $\begin{array}{c}\text { Bürste? } \\
\text { Nagelrein. }\end{array}$ & $\begin{array}{c}\text { Gewachsene } \\
\text { Kolonien }\end{array}$ \\
\hline 1 & 22. I. 17. & St. & über 10000 & 10 & B. N. & 800 \\
\hline 2 & 22. I. 17. & St. & über 10000 & 10 & B. $\mathrm{N}$. & 2500 \\
\hline 3 & 23. I. 17. & St. & 5000 & 10 & B. N. & 200 \\
\hline 4 & 23. I. 17 . & St. & 6000 & 10 & B. N. & 600 \\
\hline 5 & 20. XI. 16 . & St. & unzählige & 10 & B. N. & unzählige \\
\hline 6 & 20. XI. 16 . & St. & unzählige & 10 & B. N. & unzählige \\
\hline 7 . & 21. XI. 16. & St. & 3000 & 10 & B. N. & 2500 \\
\hline 8 & 21. XI. 16. & St. & unzählige & 10 & B. $\mathrm{X}$. & unzählige \\
\hline
\end{tabular}

Tabelle 6 .

\begin{tabular}{|c|c|c|c|c|c|c|}
\hline \multirow{3}{*}{$\begin{array}{l}\text { la } \\
2 \mathrm{a}\end{array}$} & \multirow[b]{2}{*}{ 23. XI. 16 , } & \multirow[b]{2}{*}{ St. } & \multirow[b]{2}{*}{ unzählige } & \multicolumn{3}{|c|}{ Seifeuwaschung } \\
\hline & & & & 1 & N. & 700 \\
\hline & 23. XI, 16. & St. & unzählige & 1 & N. & über 10000 \\
\hline $3 \mathbf{a}$ & 27. XI. 16. & St. & unzählige & 1 & N. & 6000 \\
\hline $4 \mathrm{a}$ & 27. XI. 16. & St. & unzählige & 1 & $\mathrm{~N}$ & 8000 \\
\hline $5 \mathbf{a}$ & 22. XI. 16. & St. & $1000^{\circ}$ & 1 & N. & 1900 \\
\hline $6 a$ & 22. XI. 16. & St. & 650 & 1 & N. & 2500 \\
\hline $7 \mathbf{a}$ & 6. XII. 16. & St. & unzählige & 1 & N. & unzählige \\
\hline $8 a$ & 6. XII. 16. & St. & über 10000 & 1 & N. & unzählige \\
\hline
\end{tabular}

Tabelle 7.

\begin{tabular}{|c|c|c|c|c|c|c|c|c|}
\hline \multirow[b]{2}{*}{$\mathrm{Nr}$. } & \multirow[b]{2}{*}{ Datum } & \multirow[b]{2}{*}{ Person } & \multirow{2}{*}{$\begin{array}{c}\text { Bakterien- } \\
\text { aufschwemunmg }\end{array}$} & \multirow{2}{*}{$\begin{array}{c}\text { Ein- } \\
\text { reib.- } \\
\text { Dauer } \\
\text { Min. }\end{array}$} & \multirow{2}{*}{$\begin{array}{c}\text { An- } \\
\text { trock.- } \\
\text { Dauer } \\
\text { Min. }\end{array}$} & \multirow[b]{2}{*}{$\begin{array}{l}\text { Gewachsene } \\
\text { Kolonien }\end{array}$} & \multicolumn{2}{|c|}{ Gips= } \\
\hline & & & & & & & $\begin{array}{c}\text { Dauer } \\
\text { Min. }\end{array}$ & $\begin{array}{l}\text { Bürste? } \\
\text { Nagelr. }\end{array}$ \\
\hline 1 & 23. I. 17. & St. & B. prodigiosus & $2-3$ & 5 & 1 & 10 & B. $\mathrm{N}$. \\
\hline 2 & 23. I. 17. & St. & B. prodigiosus & $2-3$ & 5 & 5 & 10 & B. N. \\
\hline 3 & 29. I. 17. & St. & B. coli & $2-3$ & 5 & unzählige & 10 & B. N. \\
\hline 4 & 29. I. 17. & St. & B. coli & $2-3$ & 5 & unzählige & 10 . & B. N. \\
\hline 5 & 22. I. 17. & St. & Sarcin. aurant. & $2-3$ & 5 & unzählige & 10 & B. $\mathrm{N}$. \\
\hline 6 & 22. I. 17. & St. & Sarcin. aurant. & $2-3$ & 5 & unzählige & 10 & B. N. \\
\hline 7 & 21. XI. 16. & St. & Staphylok. aureus & 3 & 3 & unzählige & 10 & B. N. \\
\hline 8 & 21. XI. 16. & St. & Staphylok. aureus & 3 & 3 & über 10000 & 10 & B. N. \\
\hline
\end{tabular}

\section{Tabelle 7 .}

1a 28. XI. 16. St.

2a 28. XI. 16. St.

$3 a$ 7. XII. 16.

42 7. XII. 16.

$5 \mathrm{a}$ 5. XII. 16.

$6 a$ 5. XII. 16.

St.

St.

St.

St.
B. prodigiosus
B. prodigiosus.

$$
\text { B. coli }
$$$$
\text { B. coli }
$$

Sarcin.aurant.

Sarcin. aurant.

$2-3$
$2-3$
$2-3$
$2-3$
$2-3$
$2-3$

Seifen-

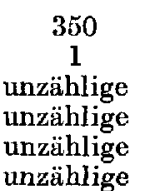

\begin{tabular}{|l|l|}
1 & $\mathrm{~N}$. \\
1 & $\mathrm{~N}$. \\
1 & $\mathrm{~N}$. \\
1 & $\mathrm{~N}$. \\
1 & $\mathrm{~N}$. \\
1 & $\mathrm{~N}$.
\end{tabular}


Gruppe 1 .

\begin{tabular}{|c|c|c|c|c|c|c|c|c|}
\hline \multicolumn{4}{|c|}{ Alkohol-Waschung } & \multirow{3}{*}{$\mid \begin{array}{c}\text { Keim- } \\
\text { abnahme } \\
\text { in } \\
\text { Prozenten }\end{array}$} & \multirow{2}{*}{\multicolumn{2}{|c|}{$\begin{array}{c}\text { Abspülen } \\
\text { der Hände. }\end{array}$}} & \multirow{3}{*}{$\begin{array}{l}\text { Wachs- } \\
\text { tums- } \\
\text { dauer } \\
\text { aller Kol. } \\
\text { Std. }\end{array}$} & \multirow{3}{*}{$\begin{array}{l}\text { Bemer- } \\
\text { kungen. }\end{array}$} \\
\hline \multirow[b]{2}{*}{$\begin{array}{l}\text { Dauer } \\
\text { Min. }\end{array}$} & \multirow{2}{*}{$\begin{array}{c}\text { Konzentr. } \\
\text { des } \\
\text { Alkohols } \\
\text { in Proz. }\end{array}$} & \multirow[b]{2}{*}{$\begin{array}{l}\text { Bürste? } \\
\text { Tupfer? }\end{array}$} & \multirow[b]{2}{*}{$\begin{array}{l}\text { Gewachs. } \\
\text { Kolonien }\end{array}$} & & & & & \\
\hline & & & & & $\begin{array}{l}\text { Dauer } \\
\text { Min. }\end{array}$ & $\begin{array}{l}\text { Gewachs. } \\
\text { Kolonien }\end{array}$ & & \\
\hline 5 & 60 & $\mathrm{~T}$. & 12 & $99 \cdot 88$ & 5 & 10 & 48 & \\
\hline 5 & 60 & $\mathrm{~T}$. & 8 & $99 \cdot 92$ & 5 & 6 & 48 & \\
\hline 5 & 70 & $\mathrm{~T}$. & 3 & $99 \cdot 94$ & 5 & 1 & 48 & \\
\hline 5 & 70 & $\mathrm{~T}$ & 8 & $99 \cdot 87$ & 5 & 1 & 48 & s. \\
\hline 5 & 80 & T. & 2 & $99 \cdot 99$ & 8 & 3 & 24 & Tab. 4. \\
\hline 5 & 80 & T. & $7 \overline{0}$ & $99 \cdot 65$ & 8 & 400 & 24 & \\
\hline 5 & 99 & T. & 20 & - & 5 & 5 & 48 & \\
\hline 5 & 99 & $\mathrm{~T}$. & 63 & $99 \cdot 68$ & 5 & 9 & 48 & \\
\hline
\end{tabular}

Gruppe 2

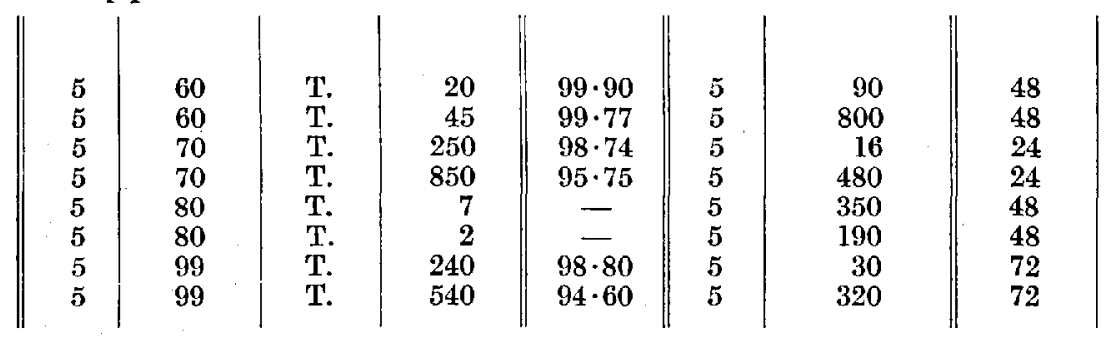

Gruppe 1.

\begin{tabular}{|c|c|c|c|c|c|c|c|}
\hline \multirow{2}{*}{$\begin{array}{c}\text { Waschung } \\
\begin{array}{c}\text { Gewachsene } \\
\text { Kolcnien }\end{array}\end{array}$} & \multicolumn{3}{|c|}{ 80proz. Alkohol-Waschung } & \multicolumn{2}{|c|}{ Abspülen der Hände } & \multirow{2}{*}{$\begin{array}{l}\text { Wachstums- } \\
\text { dauer } \\
\text { aller Kol. } \\
\text { Std. }\end{array}$} & \multirow{2}{*}{$\begin{array}{l}\text { Bemer- } \\
\text { kungen }\end{array}$} \\
\hline & $\begin{array}{l}\text { Dauer } \\
\text { Min. }\end{array}$ & $\begin{array}{l}\text { Bürste? } \\
\text { Tupfer ? }\end{array}$ & $\begin{array}{c}\text { Gewachsene } \\
\text { Kolonien }\end{array}$ & $\begin{array}{c}\text { Dauer } \\
\text { Min. }\end{array}$ & $\begin{array}{c}\text { Gewachsene } \\
\text { Kolonien }\end{array}$ & & \\
\hline $\begin{array}{c}\text { steril } \\
\text { steril } \\
\text { unzählige } \\
\text { unzählige } \\
1800 \\
2200 \\
\text { über } 10000 \\
\text { über } 10000\end{array}$ & $\begin{array}{l}5 \\
5 \\
5 \\
5 \\
5 \\
5 \\
5 \\
5\end{array}$ & $\begin{array}{l}\text { T. } \\
\text { T. } \\
\text { T. } \\
\text { T. } \\
\text { T. } \\
\text { T. } \\
\text { T. } \\
\text { T. }\end{array}$ & $\begin{array}{c}\text { steril } \\
\text { steril } \\
\text { steril } \\
4 \\
2 \\
\text { steril } \\
24 \\
34\end{array}$ & $\begin{array}{l}5 \\
5 \\
5 \\
5 \\
5 \\
5 \\
5 \\
5\end{array}$ & $\begin{array}{c}\text { steril } \\
\text { steril } \\
20 \\
18 \\
2 \\
6 \\
6 \\
13\end{array}$ & $\begin{array}{l}72 \\
72 \\
48 \\
48 \\
48 \\
48 \\
48 \\
48\end{array}$ & $\begin{array}{l}\text { s. Tab.5. } \\
\text { s. Tab.5. }\end{array}$ \\
\hline
\end{tabular}

Gruppe 2.

\section{Waschung}

unzählige

steril

unzählige

unzählige

unzählige

unzählige

\begin{tabular}{|l|}
5 \\
1 \\
5 \\
5 \\
5 \\
5 \\
5 \\
5
\end{tabular}

T.
T.
T.
T.
T.
T.

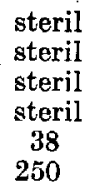

steril
steril
55
20
650
650


Operationen durch die Berïhrung mit Blut oder Körper- und Gewebstlüssigkeiten leicht aufquellen, und daB dabei bisher versteckt sitzende Bokterien mobilisiert und an die Oberfläche der Hand gelangen können, ist klar. Es blieb nun festzustellen, ob nach der Gochtschen Desinfektion weniger Keime frei werden oder bei einer Alkoholdesinfektion, der nur eine kurze Seifenwaschung vorausgeht. Oder mit anderen Worten, ist es günstiger, schon vor der Alkoholbehandlung die in den Tiefen der Haut steckenden Bakterien aufzuwühlen und die Həutporen zu öffnen, oder die in der Tiefe der Haut steckenden Bakterien an Ort und Stelle zu belassen und sie nur zu fixieren? Um diese Frage beantworten zu können, hielt ich die Hände, nachdem sie entweder mit Gips und Alkohol oder nur mit Alkohol nach 1 Minute langer Seifenwaschung desinfiziert, und der augenblickliche Keimgehalt ihrer Oberfläche ermittelt worden war, mindestens 5 Minuten lang in steriles, warmes Wasser und brachte, indem ich jede Hond für sich darin bewegte, so die Haut zum Aufquellen. Dann folgte eine Beimpfung der Agarplatten in der üblichen Weise. Zweifelsohne gehen auch in das Handbadewasser eine Anzahl Keime über, und man könnte zum Vergleiche die Menge dieser ermitteln. Doch aus der bereits genannten Arbeit von Paul und Sarwey ist es ersichtlich, daß der Keimgehalt der gebadeten Hand größer ist als der des benutzien Wassers; aus diesem Grunde nahm ich die Abimpfung von jeder Hand vor.

Der Versuchsanordnung entsprechend zerfällt die zunächst zu erörternde Tabelle 6 in zwei Teile, von denen der eine die Gips-Alkoholdesinfektion, der andere die oberflächliche Seifenwaschung-Alkohol-Methode behandelt. Wic aus der Tabelle hervorgeht, wurde zu jedem Versuche eine andere Alkoholkonzentration gewählt und zwar von 60prozentigem aufwärts bis zu absolutem. Bei genauerem Studium der Tabellen ersieht man, daß der oberflächliche Keimgehalt der Tageshand sich zu dem der nach der Gochtschen Methode desinfizierien Hand durchschnittlich wie 10000:16 verhält, während dieses Verhältnis bei Anwendung der Alkoholdesinfektion im Anschluß an eine flüchtige Seifenwaschung 10000:174 beträgt. Schon angesichts dieser Zahlen muß man die Uberlegenheit einer intensiven Vorbehandlung der Hände, speziell die der Gips-Heiswasserwaschung gegenüber einer kurzen Seifenwaschung anerkennen. Am meisten interessierte aber hier die Anzahl der Keime nach der Warmwasser-Einwirkung. Von den 8 Fällen der ersten Gruppe ist die Keimzahl in 6 Fällen geringer als die nach der Alkoholdesinfektion, in den übrigbleibenden zwei Fällen 5 und 6 dagegen hat eine Vermehrung stattgefunden.

Wie steht es in dieser Hinsicht mit den Resultaten der zweiten Gruppe? Hier ist in 4 Fällen eine Verminderung, in weiteren 4 Fällen eine Vermehrung 
der Keime eingetreten. Und zwar war dieses Verhalten der aufquellenden Wirkung des Wassers gegenüber nicht etwa direkt von der Konzentration des Alkohols abhängig, so daß vielleicht-der prozentualiter höher stehende Alkohol eine bessere Fixation der Bakterien hervorgerufen hätte; denn eine Abnahme der Bakterienzahl nach dem Handbade findet sich anschließend an die Desinfektion mit 70 Proz. und absolutem Alkohol, wogegen bei Anwendung von 60 und 80 prozentigem Alkohol eine sehr erhebliche Zunahme der oberflächlichen Handkeime nach dem Wasserbade eintrat. In Fall 1a und $2 a$ betrug diese Keimvermehrung das $4 \frac{1}{2}$ bzw. fast 18 fache und in Fall 5a und 6a sogar das 50 bzw. 95 fache. Es hat also hier in der Hälfte der Fälle die Desinfektion nicht tief genug gewirkt, während bei der GipsAlkohol-Methode nur ein wirklich schlechtes Resultat zu verzeichnen war.

Abgesehen von diesem Verhältnis, von 4 schlechten Ergebnissen zu einem einzigen, muß noch dem Umstand Rechnung getragen werden, daß sich, soweit es sich um diese ungünstigen Resultate handelt, nach Anwendung der abgekürzten Ahlfeldschen Methode die Zahlen der oberflächlichen Keime nach dem Wasserbade um das durchschnittlich mehr als 40 fache, bei der Desinfektion nach Goch $t$ dagegen um das noch nicht 6 fache vermehrt haben. Diese Keimzunahme war natürlich von der Menge der Bakterien abhängig, die sich trotz der Desinfektion noch in den tieferen Hautschichten aufhielten und durch die aufquellende und porenöffnende Wirkung des warmen Wassers an die Oberfläche gelangten. War also die Keimvermehrung nur unbedeutend, so konnte auch aer Keimgehalt der Poren und tieferen Epidermislagen nicht groß sein, oder es waren mit anderen Worten die sich in der Tiefe massenhaft aufhaltenden Bakterien durch die Desinfektion größtenteils entfernt oder vernichtet worden. Diese Tiefenwirkung ist ausgesprochener bei der Gips-Alkohol-Desinfektion als bei der abgekürzten Ahlfeldschen Methode. Das Fazit also, das aus diesen letzten Untersuchungen und ihren Ergebnissen gezogen werden muß, ist das, daß die Desinfektion nach Gocht den Keimgehalt sowohl der Oberfläche als auch der tieferen Schichten der Hand in bei weitem höheren Maße herabsetzt, als dies die vereinfachte Ahlfeldsche Methode zu tun imstande ist.

Auch die Resultate, die in Tabelle 7 zusammengestellt sind, liefern eine neue Bestätigung der eben besprochenen Versuchsreihe. Die betreffenden Versuche basieren wieder auf der künstlichen Händeinfektion, die in derselben Weise und mit denselben Bakterien ausgeführt wurde wie die frïheren. Es wurde 80prozentiger Alkohol angewandt; sonst wurde die gleiche Versuchsanordnung und Einteilung wie bei den letzten Untersuchungen gewählt. Am deutlichsten zeigt sich die Überlegenheit der Gochtschen Desinfektion bei den Untersuchungen nach einer künstlichen Infektion 
Tabelle

\begin{tabular}{|c|c|c|c|c|c|c|}
\hline \multirow{2}{*}{ Nr. } & \multirow{2}{*}{ Datum } & \multirow{2}{*}{ Person } & \multirow{2}{*}{$\begin{array}{l}\text { Kolonien } \\
\text { von der } \\
\text { Tageshand }\end{array}$} & \multicolumn{3}{|c|}{ Sehwerspatwaschung } \\
\hline & & & & $\begin{array}{l}\text { Dauer } \\
\text { in Min. }\end{array}$ & $\begin{array}{c}\text { Bürste? } \\
\text { Nagelrein. }\end{array}$ & $\begin{array}{c}\text { Gewachsene } \\
\text { Kolonien }\end{array}$ \\
\hline $\begin{array}{l}1 \\
2 \\
3 \\
4 \\
5 \\
6\end{array}$ & $\begin{array}{l}\text { 10. I. } 17 . \\
\text { 10. I. } 17 . \\
11 . \text { I. } 17 . \\
\text { 11. I. } 17 . \\
29 . \text { I. } 17 . \\
29 . \text { I. } 17 .\end{array}$ & $\begin{array}{l}\text { St. } \\
\text { St. } \\
\text { St. } \\
\text { St. } \\
\text { St. } \\
\text { St. }\end{array}$ & $\begin{array}{c}\text { über } 10000 \\
\text { unzählige } \\
3000 \\
\text { über } 10000 \\
\text { über } 10000 \\
\text { unzählige }\end{array}$ & $\begin{array}{l}10 \\
10 \\
10 \\
10 \\
10 \\
10\end{array}$ & $\begin{array}{l}\text { B. N. } \\
\text { B. N. } \\
\text { B. N. } \\
\text { B. N. } \\
\text { B. N. } \\
\text { B. N. }\end{array}$ & $\begin{array}{c}\text { unzählige } \\
\text { unzählige } \\
\text { unzählige } \\
\text { unzählige } \\
\text { über lo000 } \\
\text { unzählige }\end{array}$ \\
\hline
\end{tabular}

mit Sarcina aurantiaca. In beiden Versuchsgruppen wurde die künstliche Infektion der Hände gleich intensiv ausgeführt. Die Ergebnisse sowohl vor als auch nach dem Wasserbade weisen ganz beträ.chtliche Unterschiede zugunsten dẹr Gips-Alkohol-Methode auf. Auch der schon in Tabelle 5 angeführte Staphylokokkus-Versuch gibt einen weiteren Beweis für die Tiefenwirkung dieses Desinfektionsmodus.

Aus den letzten Untersuchungen (Tab. 6 und 7) tritt also die Utberlegenheit einer intensiven Vorbehandlung der Hände, speżiell die der GipsHeißwasser-Waschung, vor einer nur oberflächlichen Reinigung, nämlich der Alkoholdesinfektion, deutlich zutage. Ob und in welchem Umfange die Gochtsche Desinfektion in bezug auf Tiefenwirkung bessere Erfolge aufweist als die allgemein gebräuchliche Ahlfeldsche Methode, die eine gründliche Seifenwaschung unter Benutzung der Bürste in sich schließt, habe ich einer genaueren Untersuchung nicht unterzogen, doch ist aus meinen Untersuchungsergebnissen als sicher anzunehmen, daß die Desinfektion der tieferen Həutschichten nach einer voraufgegangenen Gipswaschung besser ist als nach einer auch noch so gründlichen Seifenwaschung, da zweifellos die erstere eine intensivere Auflockerung der Epidermis erzeugt als letztere.

Ich komme jetzt noch einmal auf die Untersuchungen mit anderen pulverisierten Substanzen, die möglicherweise auch bei der mechanischreinigenden Vorbehandlung der Hände hätten Verwendung finden können. Es sind die schon angedeuteten Versuche mit Schwerspat oder. Baryumsulfat, das bekanntlich in der Röntgenologie als Kontrastmittel gebraucht wird. Zuerst überzeugte ich mich durch mehrfache Kulturversuche, daß der im Handel käufliche Schwerspat vollkommen keimfrei ist. Die Händedesinfektion wurde mit diesem Mittel in derselben Weise wie mit dem Gipspulver vorgenommen.

Nach den in der Tabelle 8 aufgezeichneten Resultaten betrug die 
8.

\begin{tabular}{|c|c|c|c|c|c|c|c|}
\hline \multicolumn{4}{|c|}{ Alkoholwaschung } & \multirow[b]{2}{*}{$\begin{array}{l}\text { Keim- } \\
\text { abnahme } \\
\text { in Proz. }\end{array}$} & \multicolumn{2}{|c|}{ Abspülen der Hände } & \multirow[b]{2}{*}{$\begin{array}{c}\text { Wachstums- } \\
\text { dauer aller } \\
\text { Koloien } \\
\text { in Stunden }\end{array}$} \\
\hline $\begin{array}{l}\text { Dauer } \\
\text { in Min. }\end{array}$ & $\begin{array}{c}\text { Konzentr. } \\
\text { des } \\
\text { Alkohols } \\
\text { in Proz. } \\
\end{array}$ & Tupfer & $\begin{array}{c}\text { Ge- } \\
\text { wachsene } \\
\text { Kolonien } \\
\end{array}$ & & $\begin{array}{l}\text { Dauer } \\
\text { in Min. }\end{array}$ & \begin{tabular}{|c|} 
Ge- \\
wachsene \\
Kolonien \\
\end{tabular} & \\
\hline $\begin{array}{l}5 \\
5 \\
5 \\
5 \\
5 \\
5\end{array}$ & $\begin{array}{l}60 \\
60 \\
70 \\
70 \\
80 \\
80\end{array}$ & $\begin{array}{l}\text { T. } \\
\text { T. } \\
\text { T. } \\
\text { T. } \\
\text { T. } \\
\text { T. }\end{array}$ & $\begin{array}{r}120 \\
130 \\
20 \\
8 \\
6 \\
3\end{array}$ & $\begin{array}{l}98 \cdot 80 \\
99 \cdot 35 \\
- \\
99 \cdot 92 \\
99 \cdot 94 \\
99 \cdot 98\end{array}$ & $\begin{array}{l}5 \\
5 \\
5 \\
5 \\
5 \\
5\end{array}$ & $\begin{array}{r}120 \\
25 \\
26 \\
4 \\
6 \\
15\end{array}$ & $\begin{array}{l}48 \\
72 \\
72 \\
48 \\
48\end{array}$ \\
\hline
\end{tabular}

durchschnittliche Keimabnahme an der Handoberfläche 99.6 Proz., aber auch die Tiefenwirkung war recht zufriedenstellend, wie aus der nach dem Handwasserbade gefundenen Keimzahl hervorgeht. In keinem Falle ist eine Zunahme derselben zu beobachten. Diese Wirkungsweise des Schwerspats und die erzielten Resultate machen es doch zum mindesten höchstwahrscheinlich, daß wir es im Baryumsulfat mit einem Mittel zu tun haben, das dem Gipspulver zur vorbereitenden Händereinigung gleichwertig ist. Es besitzt außerdem den kleinen Vorzug vor letzterem, daß es beim Waschen noch angenehmer für die Haut ist. Auch praktisch hat sich der Schwerspat bereits bewährt; denn die nach einer Desinfektion mit ihm z. T. recht langdauernd vorgenommenen Operationen in der Gochtschen Klinik haben stets eine glatte, durch keine Zufälle komplizierte Wundheilung ergeben.

Vergleicht man nun die durch die bakteriologischen Versuche gewonnenen Ergebnisse der ganzen Arbeit mit einander, so ergibt sich die Antwort auf die gleich am Anfang gestellte Frage, ob die in der Praxis sich glänzend bewährte Gips-Alkohol-Händedesinfektion auch kritischen, vergleichenden Laboratoriumsuntersuchungen standhalten werde, ganz von selbst.

Nach diesen ist die Gips-Alkohol-Methode der allgemein verbreiteten Ahlfeldschen Desinfektionsweise völlig ebenbürtig, ja vielleicht sogar, was die Vernichtung der tiefsitzenden KKeime anbetrifft, dieser überlegen. Das Gleiche läßt sich auch von der Schwerspat-Alkohol-Desinfektion an Hand der zwar wenigen, aber gut ausgefallenen Versuche in Analogie mit der Gips-Alkohol-Methode und auf Grund der praktischen Erfolge behaupten. Die Vorzüge der beiden von Gocht angegebenen Händedesinfektionsmethoden gegenüber der Seifenwaschung bestehen, ganz abgesehen von ihrer bakteriologischen Wirkungsweise cinmal in der Schonung der Hände, zum anderen in ihrer Billigkeit und drittens - und das ist heutzutage das Wichtigste - in ihrer leichten Beschaffbarkeit. Uber den ersten Punkt 
habe ich mich schon weiter oben geäußert. Die schwere Beschaffung der Seife in der Kriegszeit war einer der Gründe, weshalb Gocht anstelle der Seifenwaschung die Gipsmethode setzte. ${ }^{1}$ Was den Vorzug der Billigkeit anbelangt, so will ich nur darauf hinweisen, daß bei den heutigen Preisen die übliche Seife ungeführ 12 bzw. 5mal so teuer ist als Gips oder Schwerspat, wobei ich bemerken muß, daß man mit sehr geringen Mengen dieser Substanzen auskommt. Was dieser Preisunterschied bedeutet, das werden die Krankenhäuser und Lazarette, in denen viel operiert wird, wohl zu würdigen wissen.

\section{Zusammenfassung.}

1. Die Gochtsche Händedesinfektion besteht in 10 Minuten langem Waschen in fließendem warmem Wasser mit Gips bzw. Schwerspatpulver unter Gebrauch von Bürste und Nagelreiniger und darauf folgender Desinfektion mit 70prozentigem Alkohol mit Mulltupfer.

2. Die Händedesinfektion nach Gocht unterscheidet sich von der nach AhIfeld durch die intensivere mechanische Vorbehandlung der Hände vor der Alkoholwaschung bei gleichzeitiger größerer Schonung der Hände.

3. Die Keimzahl der Handoberfläche nimmt nach der Gochtschen Desinfektion um 99.85 Proz., nach der Ahlfeldschen Desinfektion nur um 99.6 Proz. ab; auch künstlich auf der Haut verriebenen, z. T. höchst virulenten und resistenten Bakterien gegenüber hat die erstere sich als desinfektionstüchtig erwiesen.

4. Die Desinfektion nach Gocht hat sich also in der Praxis wie im bakteriologischen Versuche bewährt und ist aus diesen wie aus Gründen der Sparsamkeit und der stets leichten Beschaffung des Materials sehr zu empfehlen.

Zum Schluß ist es mir ein dringendes Bedürfnis, Hèrn Professor Dr. Gocht, Direktor des Universitätsinstitutes für Orthopädie zu Berlin, für die liebenswürdige thberlassung und Anregung zur Arbeit, sowie Herrn Privatdozenten Dr. Schürmann, stelfvertr. Direktor des Hygienischen. Institutes zu Halle, für seine vielfachen Unterstützungen und Anleitungen bei den Versuchen meinen verbindlichsten Dank auszusprechen.

1 Alabaster-Gips-Pulver (Cale. sulf. usta in feinster Form) kann von der Gips- Industrie E. Vogel in Nordhausen bezogen werden. 


\section{Literaturverzeichnis.}

Ahlfeld, Die Desinfektion des Fingers und der Hand vor geburtshilflichen Untersuchungen und Eingriffen. Deutsche med. Wochenschrițt 1895. Nr. 51.

Ahlfeld u. Vahle, Die Wirkung des Alkohols bei der geburtshilflichen Desinfektion. Ebenda 1896. Nr. 6.

Börnstein, Versuche über die Möglichkeit, infizierte Hände durch einfache Verfahren zu desinfizieren. Diese Zeitschrift. Bd. LXXIX.

v. Brunn, Neuere Bestrebungen zur Verbesserung der Vereinfachung der Hautdesinfektion. Münchener med. Wochenschrift 1908. Nr. 17.

Bürgi, Chemische Desinfektionslehre. Kolle-Wassermann. Bd. III. 1913. 2. Aufl.

Flügge, Einige Vorschläge zur Verbesserung von Desinfektionsvorschriften. Diese Zeitschrift Bd. L.

Derselbe, Grundriß der Hygiene. 1915. 8. Aufl.

Fürbringer u. Freyhan, Neue Untersuchungen über dic Desinfektion der Hände. Deutsche med. Wochenschrift 1897. S. 6.

Gocht, Händedesinfektion ohne Seife. Ebenda 1916.

Gotschlich, Desinfektionslehre. Kolle-Wassermann. Bd III. 1913. 2. Aufl.

Hansen, Über die tötende Wirkung des Äthylalkohols auf Bakterien und Hefen. Centralbl. f. Bakt. 1. Abt. Bd. XLV. 1908.

Heck, Prüfung der Wirksamkeit neuerer Alkohol-Desinfektionsmethoden der Hände in Laboratoriumsversuchen und bei Operationen. Arbeiten aus d. Institut zur Erforschung der Infektionskrankheiten in Bern. 3. Heft.

Herff, Über den Wert der Heißwasseralkoholdesinfektion für die Geburtshilfe usw. Ebenda 1906. 30.

Dersel be, Wie ist der zunehmenden Kindbettfiebersterblichkeit zu steuern usw. ? Ebenda 1907. 21.

Kolle-Hetsch, Experiment. Bakteriologie u. Injektionskrankheiten. Bd. I. 1911. 3. Aufl.

Krönig u. Blumberg, Vergleichende Untersuchungen über den Wert der mechanischen und Alkoholdesinfektion der Hände usw. Münchener med. Wachenschrift. 1900. Nr. 29/30. 
Küster, Vergleichende Untersuchungen über die Wirkung neuerer Händedesinfektionsmethoden. Arbeiten aus d. Kais. Gesundheitsamt. Bd. XLVIII.

Mikulicz, Die Desinfektion der Haut und Hände mittels Seifenspiritus. Deutsche med. Wochenschrift 1899. 24.

Paul u. Sarwey, Experimentaluntersuchungen über Händedesinfektion. Münchener med. Wochenschrift. 1900. Nr. 28-30.

Seitz, Über Händeinfektion und -desinfektion. Centralbl. f. Bakteriologie. Bd. XXXVII.

Speck, Hygienische Händedesinfektion. Diese Zeitschrift. Bd. L.

Winkler, Dissertation. Marburg 1899. 